\title{
Control de velocidad de un ventilador de una CPU
}

\author{
I. Alvarado. \\ J.A. Borja. \\ R. Haes. \\ D. Muñoz de la Peña. \\ Dpto. Ingeniería de Sistemas y Automática, Universidad de Sevilla. \\ Avd. de los Descubrimientos, s/n, \\ Sevilla 41092, ialvarado@us.es, jaborja@us.es, richard9661h@gmail.com,dmunoz@us.es,
}

\section{Resumen}

En este artículo se presenta un sistema de control minimalista que puede estar al alcance de todos los alumnos. Este sistema presenta un reto multidisciplinar en el que los alumnos necesitan aplicar conocimientos de electrónica, programación, tratamiento de señales y control. Mediante el montaje y el control de este sencillo equipo, que consta solo de cuatro componentes, los alumnos aprenderán los elementos básicos de un sistema de control.

Palabras clave: Educación, control, aprendizaje basado en proyectos, laboratorio en casa.

\section{Introducción}

Cualquier rama educativa tiene como objetivo principal dotar a los estudiantes de la capacidad de traducir los conocimientos teóricos adquiridos a lo largo de su formación en casos prácticos de la vida real. Sin embargo, no es una tarea sencilla, pues es un problema tan amplio y subjetivo, además de dependiente de las necesidades de los alumnos y de la rama del saber, que sería imposible definir un método único con el que afrontarlo.

Este reto se hace más complejo si se enfoca en la enseñanza del control de sistemas, ya que, para aplicar un algoritmo de control en un sistema real, es necesario tener conocimientos de diversas disciplinas independientes de la teoría de control, como electrónica, tratamiento y acondicionamiento de señales, programación, modelado e identificación de sistemas, uso de herramientas de simulación, etc.

Por lo tanto, en las prácticas de laboratorios de las asignaturas de control, en las que el tiempo disponible suele ser muy limitado, es típico que a los alumnos, para evitar que tengan que adquirir y aplicar los conocimientos de las disciplinas anteriormente mencionadas y puedan centrarse en la cuestión en estudio, se les proporcione un sistema totalmente simplificado y acondicionado, de forma que la única labor pendiente sea introducir el algoritmo de control en cuestión y analizar el correcto funcionamiento.
El gran problema que supone este enfoque viene cuando el alumno tiene que controlar un sistema real: aunque sabe cómo diseñar un controlador, no sabe cómo implementarlo.

Una estrategia educativa que trata de abordar este problema es el aprendizaje basado en proyectos (ABP), [7] [5]. Esta metodología consiste en abordar el problema entorno a un sistema real, resolviendo cada una de las cuestiones que se presentan para el mismo y adquiriendo el alumno el conjunto de conocimientos requeridos. Esto se consigue gracias a la investigación y colaboración de los estudiantes a lo largo del proyecto: formulando y explorando preguntas importantes y significativas, realizando hipótesis y predicciones, diseñando y analizando posibles soluciones, compartiendo ideas, etc.

No obstante, para algunos campos, y concretamente para el campo de la automática, esta solución da lugar a un nuevo problema: disponer de un sistema real para analizar y controlar.

Para solucionar esto último, una posible opción es el uso de laboratorios virtuales, ver [3] y sus referencias. El problema de estos es que suelen estar preparados para que el usuario solo tenga que introducir las directrices del ensayo que desea, pues no tiene acceso físico para abordar el problema de cero. Por lo tanto, el alumno no aprenderá a atacar a un sistema de la vida real, pues no estará acondicionado. Es decir, se tiene el mismo problema que se comentó para las prácticas de laboratorio en las que se usaban sistemas simplificados para poder centrar la práctica en la cuestión en estudio dentro del horario disponible.

Por otro lado, otra potencial solución que ha surgido recientemente, debido a la exponencial reducción del precio de los componentes electrónicos en los últimos años, es el desarrollo de plataformas de bajo costo. Por ejemplo, en [4] se presenta un robot autoequilibrado de dos ruedas, en [8] se muestra un ejemplo de una bola y una viga, en [6] se construye un levitador magnético para enseñar a controlar, y en [2] se presenta un robot móvil autoequilibrado.

En resumen, se tienen accesible todos los disposi- 
tivos necesarios a un precio tan reducido que cada alumno podría adquirir su propio equipo para trabajar fuera del centro educativo y en horario no lectivo. Es decir, cada alumno podría tener un laboratorio en su propia casa, con acceso físico y sin un horario limitado.

Este artículo presenta un sistema de control compuesto por solo 4 elementos. Un ventilador de una CPU de 4 hilos (2 de alimentación, el tercero es la salida del encoder interno y el cuarto es una señal de control de la velocidad del mismo); un potenciómetro de 10K (que usaremos para identificar el sistema primero y para proporcionar la referencia después), una fuente de alimentación de $12 \mathrm{~V}$ y cualquier Arduino. Los alumnos van a aprender a controlar la velocidad del ventilador mediante un PID. El tiempo de muestreo se garantizará mediante interrupciones temporales. Interrupciones asociadas al cambio de un pin serán usadas para medir la velocidad y además tendrán que programar un filtro para tener una medida aceptable de la velocidad (tratamiento de señales). El sistema es un sistema estable de primer orden, por lo que sintonizar el control es muy sencillo. Esta practica es interesante como un primer contacto con un sistema de control. Usamos este ejercicio como ejercicio previo a cualquier sistema de control más complejo. La figura 1 muestra el montaje resultante.

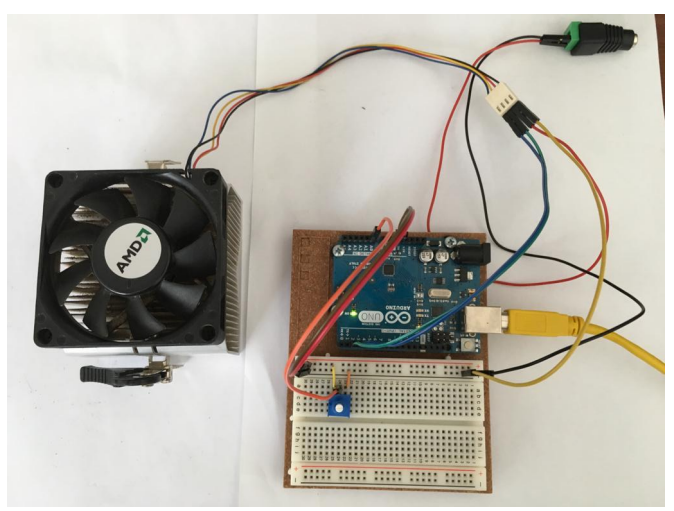

Figura 1: Fotografía del proyecto resultante

Aún siendo un sistema con una dinámica muy sencilla, este laboratorio tiene como propiedad fundamental que por su coste y por la sencillez del montaje, es posible que cada alumno adquiera su propio equipo para trabajar en casa y resolver los diferentes problemas que propongan.

Esto permite realizar prácticas con una mayor profundidad, incluyendo un análisis más completo de lo que sería posible realizar en el laboratorio en una sesión de tiempo limitada. Además el trabajo con el equipo se convierte en multidisciplinar, aplicando conocimientos no sólo de control, si no también de electrónica, programación y señales.
Este artículo se organizará de la siguiente manera; Primero, se presenta el prototipo de hardware. A continuación, se presenta el conexionado, la etapa de identificación, el esquema de control y finalmente algunos resultados. El artículo finaliza con algunas conclusiones.

\section{Hardware}

El equipo ha sido diseñado de forma que pueda ser construido por alumnos incluyendo las conexiones entre las diferentes partes del mismo. La mayoría de las partes se pueden reciclar de equipos viejos, pero, si no, se pueden conseguir por $13 € \sin$ incluir el Arduino.

En nuestro caso el ventilador es de un PC en desuso, la fuente de $12 \mathrm{~V}$ puede ser la misma del $\mathrm{PC}$ o cualquier transformador que tengamos por casa. La clave está en que el ventilador de la CPU ya viene preparado para ser controlado por una señal PWM y como esta es una señal digital, aunque el ventilador funcione a $12 \mathrm{~V}$, los $5 \mathrm{~V}$ la señal PWM del Arduino son suficientes. También tiene un encoder interno que vamos a usar para medir la velocidad, con lo que ya tenemos todo lo necesario para controlar la velocidad del mismo. A continuación se presentan, por separado, las distintas partes del mismo.

\subsection{Ventilador CPU}

Tiene que ser un ventilador de 4 hilos, si no, no es válido para esta práctica. Estos ventiladores son capaces de modificar su velocidad porque incorporan una señal PWM de control. Además, incorporan un encóder que se cierra una o dos veces por vuelta en el interior del mismo y cuya salida también es accesible para poder medir la velocidad de giro. Casi todos los ventiladores de las CPUs los incorporan. Suelen venir unidos al disipador de calor del micro. En la figura 2 puede verse el ventilador usado para este artículo.

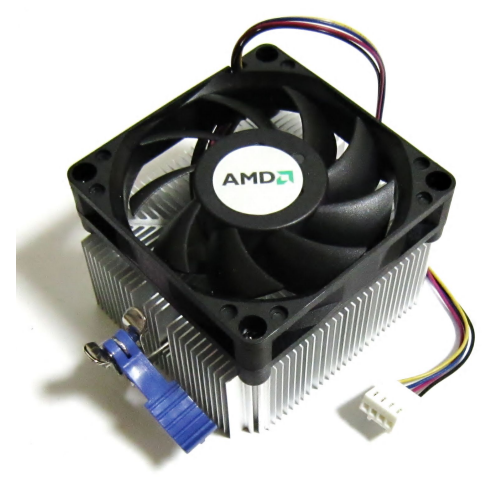

Figura 2: CPU FAN 


\subsection{Potenciómetro de $10 \mathrm{~K}$}

En la figura 3 puede verse un potenciómetro como el usado en la práctica.

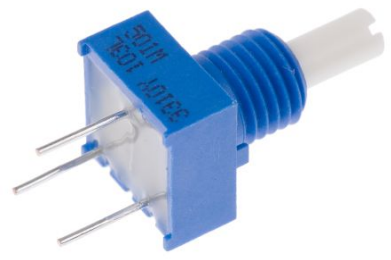

Figura 3: Potenciómetro

Utilizaremos el potenciómetro como divisor de tensión. Conectando un pin a $5 \mathrm{~V}$, el otro a GND y el pin intermedio a una de las entradas analógicas del Arduino. De esta forma tendremos un número que va de 0 a 1023 (ya que la entrada analógica del Arduino es de 10 bits) controlable por el usuario.

Esta entrada analógica será utilizada para fijar la señal de actuación en el modo de control manual y la referencia en el modo de control automático.

\subsection{Fuente de alimentación de $12 \mathrm{~V}$}

Nos vale cualquier fuente de continua de $12 \mathrm{~V}$, lo único a tener en cuenta es que se necesita un adaptador para poder conectar fácilmente la fuente al resto del circuito. En la figura 4 puede verse la fuente de alimentación usada.

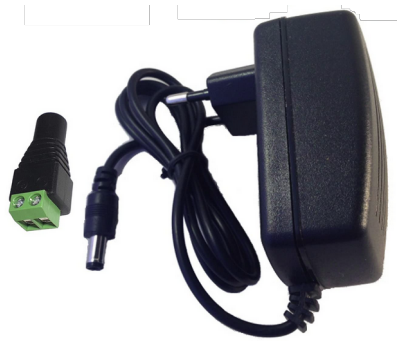

Figura 4: Fuente de alimentación y adaptador.

\subsection{Arduino}

Para este ejercicio usamos un Arduino Uno, pero puede ser cualquier Arduino. Es un microcontrolador basado en un ATmega328P. Tiene 14 pines digitales (6 de ellos PWM) y 6 entradas analógicas de 10bits. La velocidad del microcontrolador es $16 \mathrm{MHz}$. La placa contiene toda la electrónica adicional para poder usar los recursos del microcontrolador. En la figura 5 puede verse una foto de un Arduino UNO.

En este proyecto usaremos esta placa porque es la más común, y ya la tenemos de otros equipos de prácticas.

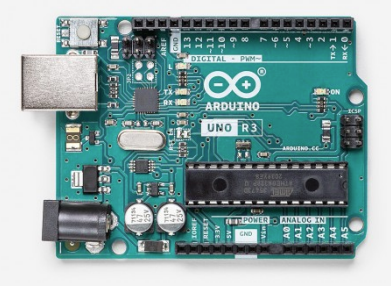

Figura 5: Arduino Uno

Vamos a usar el Arduino para procesar la información proveniente del encoder y generar la señal PWM necesaria para que el motor gire a la frecuencia de referencia determinada por el potenciómetro. Además, sacará por el puerto serie la referencia, la velocidad actual y la acción de control. Estas tres variables podrán visualizarse por medio de la herramienta Serial Plotter que trae el IDE de Arduino.

\section{Conexionado}

El circuito eléctrico a montar es muy sencillo como puede verse en la figura 6 . Es muy importante tener cuidado que el terminal con $12 \mathrm{~V}$ de la fuente de alimentación no se conecte al Arduino de ninguna manera pues podría dañarlo. También es muy importante conectar la tierra de la fuente con la del Arduino.

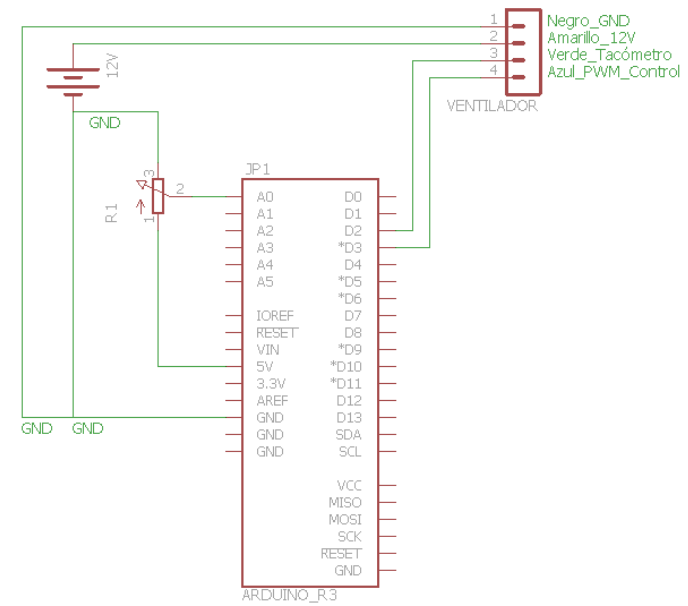

Figura 6: Conexionado

\section{Identificación}

Antes de empezar a controlar el motor, se enseña al alumno cómo trabajar con interrupciones temporales, y cómo trabajar con interrupciones asociadas al cambio de estado de un pin. Utilizaremos estas segundas para ir incrementando un contador que chequearemos cada $100 \mathrm{~ms}$ (tiempo de muestreo). Llamaremos $n p_{100}$ a esta cantidad. Aparte 
se conecta el potenciómetro y se conecta a una entrada analógica. En este primer ejercicio mapearemos la medida del potenciómetro a $[0,255]$ y aplicaremos este valor a la señal PWM del pin que controla la velocidad. Anotaremos la $n p_{100}$ mínima y $n p_{100}$ máxima.

Veremos que $n p_{100}$ solo puede coger 8 o 10 valores diferentes. Con esta precisión en el la medida es imposible hacer un control fino de la velocidad . Hace falta programar una etapa de acondicionamiento de la señal como se verá en bloque 2 de la sección 5 .

\section{Esquema de control}

El esquema de control es el típico problema de regulación en el que el objetivo de control es mantener la velocidad de giro del motor igual a la de la referencia actuando sobre el motor mediante una señal PWM. En la figura 7 vemos los bloques a programar:

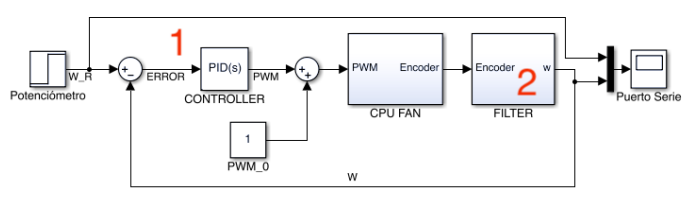

Figura 7: Esquema de control

El bloque 1, es el controlador PID, que se va a ejecutar cada $100 \mathrm{~ms}$. Recibe el error de seguimiento y genera $\triangle P W M=P W M-P W M_{0}$, Siendo $P W M_{0}$ El valor de la PWM en el punto de trabajo y $\triangle P W M$ la salida del PID que es el incremento de la señal PWM respecto al punto de trabajo.

El bloque 2 construirá la velocidad de giro del motor $\omega$ a partir de $n p_{100}$. La idea es crear un buffer que almacene las 10 últimas medidas de $n p_{100}$. En cada periodo de muestreo se añadirá una medida nueva y se desechará la más antigua. En cada periodo de muestreo se sumarán los valores de las 10 medidas del buffer y esa será la medida de $\omega$ es decir las revoluciones por segundo.

$$
\omega(k)=\sum_{i=0}^{i=9} n p_{100}(k-i)
$$

\section{Resultados docentes}

Este equipo de prácticas se ha usado como ejercicio de iniciación al control con Arduino en múltiples ocasiones. Se empezó usando con los alumnos que realizaban los proyectos de fin de grado y de fin de master relacionados con la construcción de algún dispositivo de control basado en Arduino.
Es un ejercicio sencillo que asienta muy bien las bases de cualquier sistema de control.

Sorprendentemente lo que más les cuesta de este ejercicio es programar el buffer y entender cómo funciona la función map de Arduino (que hace un mapeo de una variable en un domino a otro dominio).

El año académico 2020-21 se usó como entrenamiento de los alumnos que hicieron el proyecto voluntario de la asignatura de Control Automático del grado en Ingeniería de Telecomunicaciones que consistía en la realización de un robot siguelíneas que ha dado lugar a otra publicación en este mismo congreso [1].

Los resultados han sido buenos y el año académico 2021-22 dicho proyecto pasa a ser obligatorio en la misma asignatura, aunque ha habido que adaptar el currículo para dar cabida al mismo.

\section{Resultados del control}

A continuación se presentan los resultados obtenidos. En las figuras 8 y 9 puede verse una captura de la herramienta Serial Plotter de Arduino mostrando la evolución de la referencia, de la velocidad del motor y de la señal PWM que controla el motor.

En la figura 8 puede verse como se comporta el sistema ante varios cambios en la referencia.

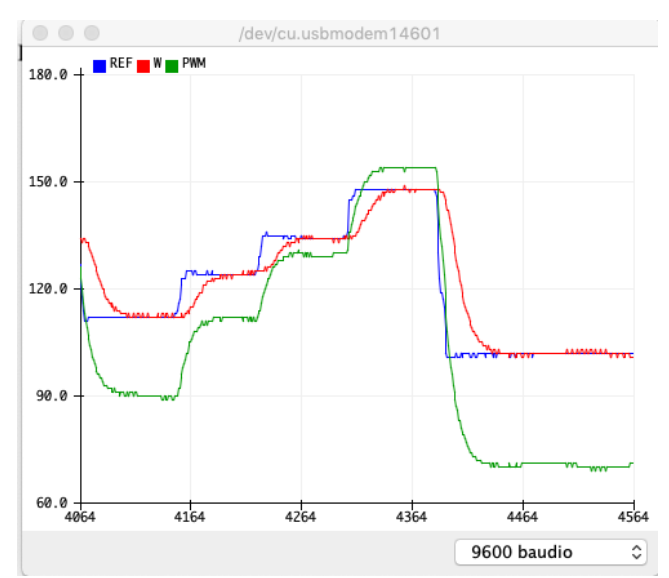

Figura 8: Gráfica con los resultados del control en seguimiento

En la figura 9 puede verse la respuesta del controlador cuando se somete al sistema a una perturbación, en concreto, se pone una hoja de papel encima del ventilador obstaculizando el paso del aire. Puede verse como la velocidad se mantiene y como la acción de control varía para compensar el efecto de la perturbación. En la última parte del ensayo se vuelve a retirar la hoja de papel. 


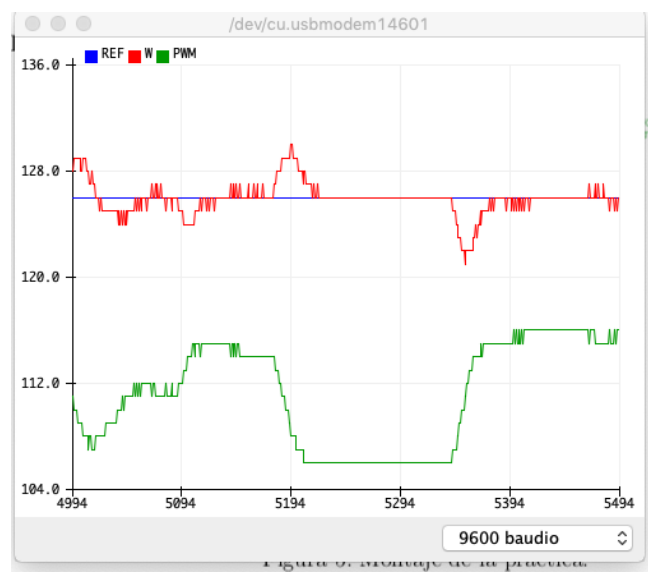

Figura 9: Gráfica con los resultados del control en regulación

\section{English summary}

\section{Speed control of a CPU fan as a first control exercise}

\begin{abstract}
This article presents a minimalist control system that can be available to everyone, including students. As all control projects are multidisciplinary projects, students need to apply knowledge of electronics, programming, signal processing, and control. By assembling and controlling this simple kit, which consists of only 4 components, students will learn the basic elements of a control system.
\end{abstract}

Keywords: Education, control, project-based learning, home lab.

\section{Referencias}

[1] I. Alvarado, J.A. Borja, F. Salas, D. Muñoz de la Peña (2021). Aprende fundamentos de control construyendo un siguelíneas. Jornadas de Automática, Castellón, España Septiembre 1-3.

[2] C. Gonzalez, I. Alvarado, D. Muñoz La Peña (2017). Low cost two-wheels self-balancing robot for control education. 20th IFAC Word Congress, Toulouse, France July 9-14.

[3] Goodwin, G.C., Medioli, A.M., Sher, W., Vlacic, L.B. and Welsh, J.S., (2011). Emulation-based virtual laboratories: a lowcost alternative to physical experiments in control engineering education. IEEE Transactions on Education, 54(1), pp.48-55.
[4] Hau-Shiue, J. and Kai-Yew, L. (2013). Design and control of a two-wheel self-balancing robot using the arduino microcontroller board. 10th IEEE International Conference on Control and Automation (ICCA), 634-639.

[5] M. Lehmann , P. Christensen , X. Du \& M Thrane (2008) Problem-oriented and projectbased learning (POPBL) as an innovative learning strategy for sustainable development in engineering

[6] Lilienkamp, K.A. and Lundberg, K. (2004). Low-cost magnetic levitation project kits for teaching feedback system design. Proceedings of the American Control Conference.

[7] Perrenet, J.C., Bouhuijs, P.A.J. and Smits, J.G.M.M., (2000) The suitability of problembased learning for engineering education: theory and practice. Teaching in higher education, 5(3), pp.345-358.

[8] Rashied, Z., Hamees, M., Hassan, M.U., Hameed, S., and Khatri, N.A. (2016). Real time implementation of Robust PID controller for stabilization of Ball Balancing Beam. International Journal of Conceptions on Information Technology and Computing. 6-9.

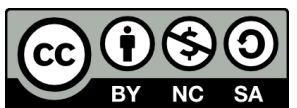

(C) 2021 by the authors. Submitted for possible open access publication under the terms and conditions of the Creative Commons Attribution CC BY-NC-SA 4.0 license (https://creativecommons.org/licenses/by-ncsa/4.0/deed.es). 